\title{
Infant lumbar and thoracic epidurals for abdominal surgeries: cases in a paediatric tertiary institution
}

\author{
Sze Ying Thong ${ }^{1}$, mBBS, MMed, Eliza I-Lin Sin $^{2}$, MBBS, MRCs, Diana Xin Hui Chan ${ }^{1}$, MBBS, MMed, Jagdish M Shahani ${ }^{3}$, MBBS, MD
}

\begin{abstract}
INTRODUCTION There is strong evidence that epidural analgesia provides good postoperative pain relief in adults, but its use in infants is less established. In this retrospective study, we present our experience with managing infant epidural analgesia for abdominal surgeries in a tertiary paediatric institution.

METHODS The records of 54 infants who had received a thoracic or lumbar epidural as perioperative analgesia for abdominal surgeries were included. The mean age of the infants was 6.1 (standard deviation [SD] 3.8) months and their mean weight was $6.8 \mathrm{~kg}$ (SD 1.8). Most (63\%) had an ASA (American Society of Anesthesiologists) status of 2 and all underwent elective gastrointestinal, urogenital, hepatobiliary or retroperitoneal surgeries. 20 catheters $(37.0 \%)$ were inserted in the thoracic region and $33(61.1 \%)$ in the lumbar region.

RESULTS A total of 52 (96.3\%) catheters provided adequate intraoperative analgesia and 36 (66.7\%) provided effective analgesia for the postoperative period. Active management of epidural analgesia, such as through epidural top-ups and infusion rate adjustment, was necessary to optimise analgesia in 22 (44\%) of the 50 patients postoperatively. Reasons for premature catheter removal were mainly technical issues such as catheter disconnection, leakage and blockage.

CONCLUSION Our data suggests that in experienced hands, specialised settings and active management, the success rate of epidural analgesia in infants undergoing major abdominal surgeries is high and without major incident.
\end{abstract}

Keywords: abdominal surgery, infant epidural, postoperative analgesia

\section{INTRODUCTION}

Epidural analgesia provides excellent pain relief after surgery. In adults, the efficacy of postoperative epidural analgesia is superior to that of parenteral opioids regardless of the analgesic agents used and site of catheter placement. ${ }^{(1)}$ However, there are considerations that limit the use of epidural analgesia in children, particularly infants. It has been difficult to reach a consensus on the risk-benefit ratio of the technique due to imprecise pain assessment tools for this age group, perceived technical difficulty, safety concerns in establishing and maintaining epidural infusion, and logistical issues.

Previously, the main role of regional anaesthesia in infants was to allow sick infants who were high-risk for general anaesthesia to avoid it. Although research has demonstrated advantages of neuraxial anaesthesia in combination with general anaesthesia in infants, such as the volatile and opioid-sparing effects, earlier tracheal extubation and improved postoperative analgesia, there is a lack of research on the efficacy of lumbar and thoracic epidurals as intraoperative and postoperative analgesia for infants. ${ }^{(2)}$

In the present study, we describe our experience managing infant epidural analgesia for abdominal surgeries in KK Women's and Children's Hospital, a 830-bed tertiary hospital for women and children in Singapore. For major abdominal surgeries where significant perioperative stress and intense pain are anticipated, the institution recommends epidural analgesia for optimal pain management, facilitation of postoperative extubation and reduction of narcotic requirements. Objective data on the efficacy and complications of epidural analgesia would allow us to evaluate the risks and benefits of this technique.

\section{METHODS}

Information on patients aged $<1$ year who had received an epidural for postoperative analgesia after abdominal surgeries was retrieved from the paediatric anaesthesia department's database of all cases performed. Patients who had caudal analgesia or surgeries that did not involve abdominal incisions were excluded. The retrospective study was approved by the SingHealth Centralised Institutional Review Board.

For all procedures, after informed consent was obtained, general anaesthesia was induced in the patients via an intravenous (IV) or inhalational technique under standard monitoring. This included a minimum of continuous 3-lead electrocardiography; pulse oximetry; monitoring of end-tidal carbon dioxide, fraction of inspired oxygen, minimum alveolar concentration of volatile agents, respiratory rate, airway pressure and tidal volume; and intermittent noninvasive blood pressure measurements. Each patient was placed in the lateral position with neck, hips and knees flexed for epidural catheter insertion prior to surgery. A specialist paediatric anaesthetist performed the epidural insertion under full sterility. Most epidurals were inserted using the landmark technique. If ultrasonography was used, the T12 vertebra, and hence the other thoracic and lumbar vertebrae, was identified by locating the last rib.

The catheter insertion site was chosen so as to locate the tip of the catheter at the midpoint of the vertebral segments to be blocked. This was typically at the lowest suitable intervertebral level of the segment. Some practitioners may choose to insert the Tuohy needle at a lower vertebral level (i.e. lumbar region) and thread the catheter towards the thoracic level to mitigate

${ }^{1}$ Department of Anaesthesia, ${ }^{2}$ Department of General Surgery, Singapore General Hospital, ${ }^{3}$ Department of Paediatric Anaesthesia, KK Women's and Children's Hospital, Singapore Correspondence: Dr Sze Ying Thong, Consultant, Department of Anaesthesia, Singapore General Hospital, Block 2 Level 2 , Outram Road, Singapore 169608. thongszeying@gmail.com 


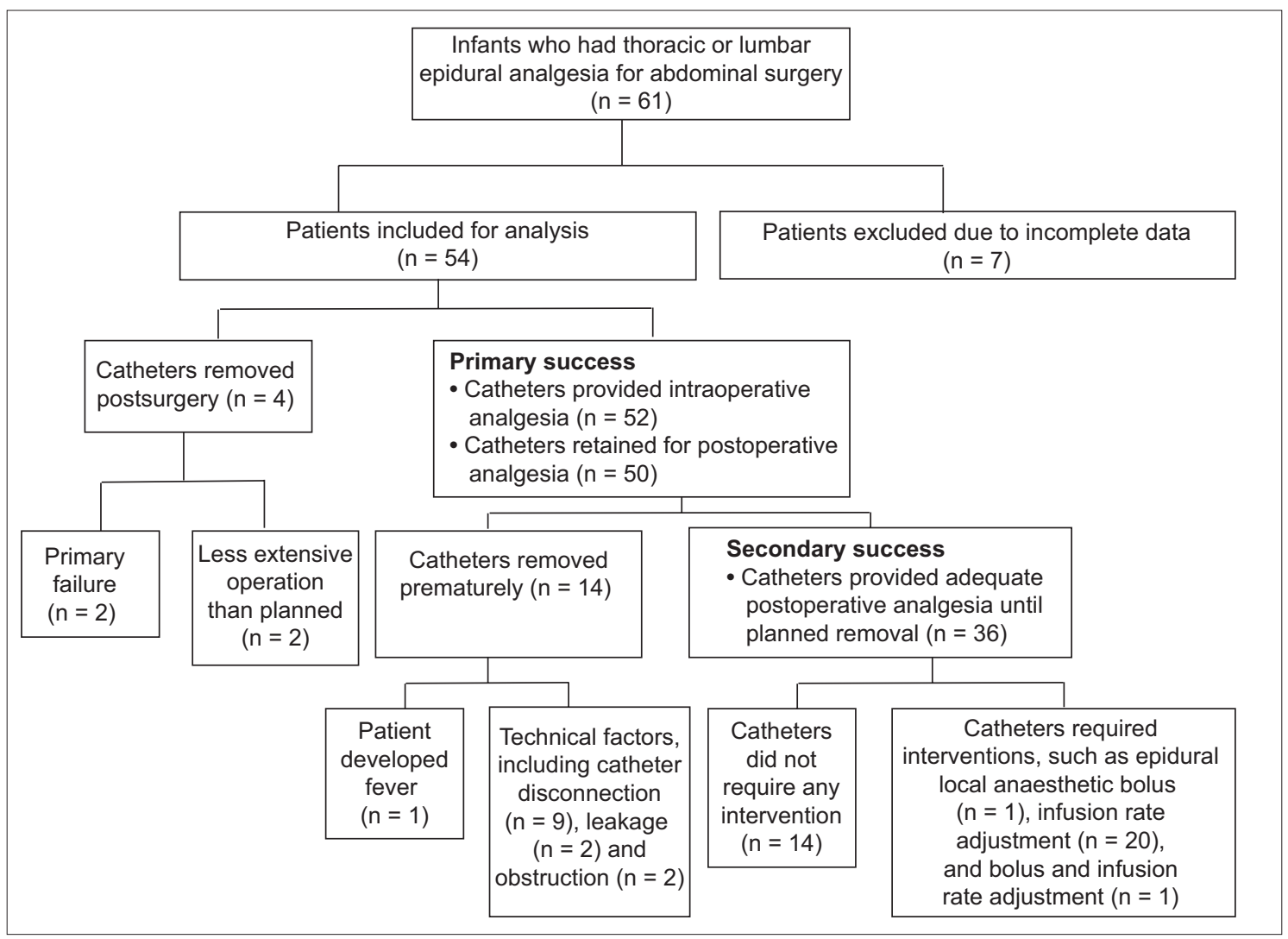

Fig. 1 Flow chart shows patient outcomes of epidural analgesia.

the risk of neurological injury in case of needle misadventure. Paediatric epidural catheter sets with a 19-gauge Tuohy needle and 23-gauge catheter were recommended by the institution. Depth from the skin to the ligamentum flavum, in millimetres, was estimated using a formula $(1.5 \times$ body weight in $\mathrm{kg})$. Continuous loss-of-resistance technique with saline or air was used to locate the epidural space.

Department protocol recommended the use of a maximum dose of $0.25 \%$ bupivacaine $0.7 \mathrm{~mL} / \mathrm{kg}$ bolus to establish initial epidural blockade at the start of surgery. Epidural analgesia was then maintained with infusions using $0.1 \%$ bupivacaine at doses below the maximum of $0.2 \mathrm{mg} / \mathrm{kg} / \mathrm{h}$ for intraoperative analgesia. Fentanyl was not routinely added to the regimen in infants aged $<6$ months due to increased risk of respiratory depression. Changes in haemodynamic variables in response to surgical stimulation were observed as a measure of epidural analgesia efficacy. IV opioids were administered for rescue analgesia if the maximum dosage of local anaesthetics was reached. After surgery, all patients were extubated in the operating theatre and observed in the recovery room. They were then discharged to the high dependency unit (HDU), which had a nursing ratio of one nurse to one or two patients. Patients' vital signs were monitored hourly and continuous pulse oximetry was done if necessary.

Patients were assessed for pain using the Face, Legs, Activity, Cry and Consolability (FLACC) scale. ${ }^{(3)}$ Sensory levels were not assessed. Additional analgesia in the form of a top-up of epidural local anaesthetic and/or IV opioids was administered if required. Acetaminophen and ibuprofen were added where possible. An acute pain service team led by a paediatric consultant anaesthetist assessed patients twice a day and provided 24-hour on-site assistance. Epidural analgesia was considered successful if it was (a) successfully sited and provided adequate intraoperative analgesia (primary success) and (b) provided adequate analgesia in the postoperative period (secondary success).

Data was analysed using the Statistical Package for the Social Sciences version 16.0 (SPSS Inc, Chicago, IL, USA). Categorical data was presented as numbers and percentages, and compared using the chi-square test. Continuous variables were presented as mean and standard deviation (SD). Statistical significance was set at $\mathrm{p}$-value $<0.05$.

\section{RESULTS}

Sixty-one patients received epidural analgesia from 13 August 1997 to 30 April 2014. Seven patients were excluded because their medical records were incomplete and data from 54 patients was analysed. Patients' demographic and epidural data are presented in Tables I \& II. Their outcomes are shown in Figure 1.

Intraoperatively, four patients received IV morphine, two patients received IV fentanyl infusion and one patient received IV remifentanil infusion. The two patients who received IV fentanyl infusion did not have working epidural catheters and the epidural catheters were removed at the end of the surgery. All patients were successfully extubated and did not require observation in the intensive care unit. In the recovery unit, three patients required a bolus epidural top-up of local anaesthetic. All the patients who required IV morphine or remifentanil intraoperatively $(n=5)$ or epidural top-up $(n=3)$ in the recovery unit were managed adequately with epidural analgesia in the HDU for the next 
Table I. Patient characteristics and operation details $(n=54)$.

\begin{tabular}{|c|c|}
\hline Characteristic & No. (\%) \\
\hline Age* (mth) $^{*}$ & $6.1 \pm 3.8$ \\
\hline \multicolumn{2}{|l|}{ Age group (mth) } \\
\hline Neonate $(<1)$ & 0 \\
\hline $1-6$ & $26(48.1)$ \\
\hline $6-12$ & $28(51.9)$ \\
\hline Weight* (kg) & $6.8 \pm 1.8$ \\
\hline \multicolumn{2}{|l|}{ Gender } \\
\hline Male & $27(50)$ \\
\hline Female & $27(50)$ \\
\hline \multicolumn{2}{|l|}{ ASA score } \\
\hline 1 & $9(16.7)$ \\
\hline 2 & $34(63.0)$ \\
\hline 3 & $10(18.5)$ \\
\hline Unknown & $1(1.9)$ \\
\hline \multicolumn{2}{|l|}{ Type of operation } \\
\hline $\begin{array}{l}\text { Gastrointestinal (fundoplication, surgery for } \\
\text { Hirschsprung disease, colonic stricture or } \\
\text { anorectal malformation) }\end{array}$ & $16(29.6)$ \\
\hline $\begin{array}{l}\text { Urogenital (surgery for vesicoureteric reflux, } \\
\text { duplex kidney or renal tumour excision) }\end{array}$ & $17(31.5)$ \\
\hline $\begin{array}{l}\text { Hepatobiliary (choledochal cyst excision } \\
\text { and anastomosis, Kasai procedure, } \\
\text { hepatoblastoma excision) }\end{array}$ & $17(31.5)$ \\
\hline $\begin{array}{l}\text { Others (retroperitoneal mass or } \\
\text { neuroblastoma excision) }\end{array}$ & $3(5.6)$ \\
\hline Unknown & $1(1.9)$ \\
\hline Duration of surgery* (hr:min) & $4: 06 \pm 2: 04$ \\
\hline \multicolumn{2}{|l|}{ Incision site } \\
\hline Above umbilicus & $28(51.9)$ \\
\hline Below umbilicus & $23(42.6)$ \\
\hline Above and below umbilicus & $3(5.6)$ \\
\hline
\end{tabular}

* Data presented as mean \pm standard deviation. ASA: American Society of Anesthesiologists

29-93 hours. Five of these patients had their epidural catheter removed in less than 48 hours: three patients were discharged from the HDU and two patients had disconnected catheters. Two epidural catheters were removed in the recovery room, as the patients' eventual operations were less extensive than planned and epidural analgesia was not required.

In the HDU, 19 (38\%) patients of age > 6 months had fentanyl added to their epidural local anaesthetic infusate under the discretion of the attending anaesthetist. Patients who received epidural fentanyl did not require epidural top-up or IV opioid rescue for breakthrough pain in the HDU. They were also less likely to require an increase in the infusion rate of the epidural local anaesthetic. Among the patients who required an increase, seven received epidural fentanyl and 14 patients did not. However, this was not statistically significant $(p=0.768)$. Two patients required bolus epidural top-up in the HDU to satisfactorily manage their pain. Subsequently, their epidural analgesia was satisfactory and the catheters were removed after 66 hours and 71 hours when epidural analgesia was no longer required. One patient who had undergone hepatoblastoma resection had breakthrough
Table II. Parameters of epidural insertion in study population $(n=54)$.

\begin{tabular}{|c|c|}
\hline Parameter & No. (\%) \\
\hline \multicolumn{2}{|l|}{ Epidural insertion level } \\
\hline Thoracic & $20(37.0)$ \\
\hline Lumbar & $33(61.1)$ \\
\hline Not stated & $1(1.9)$ \\
\hline \multicolumn{2}{|l|}{ Site of insertion } \\
\hline Midline & $40(74.1)$ \\
\hline Paramedian & $2(3.7)$ \\
\hline Not stated & $12(22.2)$ \\
\hline \multicolumn{2}{|l|}{ Loss-of-resistance technique } \\
\hline Saline & $26(48.1)$ \\
\hline Air & $25(46.3)$ \\
\hline Not stated & $3(5.6)$ \\
\hline \multicolumn{2}{|l|}{ Number of attempts } \\
\hline 1 & $29(53.7)$ \\
\hline 2 & $3(5.6)$ \\
\hline 3 & $1(1.9)$ \\
\hline Not stated & $21(38.9)$ \\
\hline \multicolumn{2}{|l|}{ Tuohy needle size (gauge) } \\
\hline 18 & $11(20.4)$ \\
\hline 19 & $41(75.9)$ \\
\hline 20 & $1(1.9)$ \\
\hline Not stated & $1(1.9)$ \\
\hline \multicolumn{2}{|l|}{ Catheter size (gauge) } \\
\hline 19 & $2(3.7)$ \\
\hline 21 & $20(37.0)$ \\
\hline 22 & $7(13.0)$ \\
\hline 23 & $14(25.9)$ \\
\hline 24 & $3(5.6)$ \\
\hline Not stated & $8(14.8)$ \\
\hline \multicolumn{2}{|l|}{ Depth of epidural space $(\mathrm{cm})$} \\
\hline Median (range) & $1.1(0.8-2.0)$ \\
\hline Mean \pm standard deviation & $1.3 \pm 0.3$ \\
\hline \multicolumn{2}{|c|}{ Length of catheter left in situ (cm) } \\
\hline Median (range) & $5.0(3.0-11.0)$ \\
\hline Mean \pm standard deviation & $5.2 \pm 1.8$ \\
\hline
\end{tabular}

pain and required a morphine infusion; the epidural catheter was found disconnected at the 23rd hour postsurgery. He was 7.6 months old, weighed $6.3 \mathrm{~kg}$ and had a physical status of 3 on the American Society of Anesthesiologists scale. The patient suffered transient venous oxygen desaturation to $85 \%$. Following tactile stimulation, no further complications occurred. Treatment with an opioid antagonist was not necessary.

Outcomes of the administration of epidural catheters are presented in Table III. One catheter was removed after 24 hours as the patient had a fever and it was difficult to determine if it was catheter-related; there were no symptoms or signs suggestive of catheter-related infection. Postoperative fever was relatively common, occurring in 18 (36\%) of the 50 patients during the time that the epidural analgesia was in effect. All patients who needed IV morphine intraoperatively $(\mathrm{n}=4)$ and those who had their failed epidural catheter removed in the recovery unit 
Table III. Duration of epidural analgesia and reasons for its discontinuation $(n=50)$.

\begin{tabular}{|c|c|c|c|c|c|c|c|}
\hline \multirow{2}{*}{$\begin{array}{l}\text { Duration } \\
\text { of epidural } \\
\text { infusion (hr) }\end{array}$} & \multicolumn{6}{|c|}{ Reasons for discontinuation of epidural analgesia } & \multirow[t]{2}{*}{ No. (\%) } \\
\hline & $\begin{array}{l}\text { Planned } \\
\text { removal }\end{array}$ & $\begin{array}{l}\text { Discharge } \\
\text { from HDU }\end{array}$ & Fever & $\begin{array}{c}\text { Catheter } \\
\text { disconnection }\end{array}$ & $\begin{array}{l}\text { Catheter } \\
\text { leakage }\end{array}$ & $\begin{array}{l}\text { Catheter } \\
\text { obstruction }\end{array}$ & \\
\hline $12-23$ & 0 & 0 & 0 & 1 & 0 & 0 & $1(2)$ \\
\hline $24-47$ & 7 & 4 & 1 & 6 & 2 & 2 & $22(44)$ \\
\hline $48-71$ & 18 & 1 & 0 & 2 & 0 & 0 & $21(42)$ \\
\hline 72-95 & 6 & 0 & 0 & 0 & 0 & 0 & $6(12)$ \\
\hline No. (\%) & $31(62)$ & $5(10)$ & $1(2)$ & $9(18)$ & $2(4)$ & $2(4)$ & \\
\hline
\end{tabular}

HDU: high dependency unit

$(n=2)$ received lumbar epidurals. The level of epidural catheter insertion (lumbar or thoracic) did not appear to affect the number of insertion attempts, postoperative breakthrough pain, need for infusion adjustment or premature catheter removal.

\section{DISCUSSION}

In this retrospective study, we reviewed the data of relatively healthy infants who underwent major abdominal surgeries using lumbar or thoracic epidurals as the main form of analgesia. The overall primary success rate of epidural analgesia in our series was $96.3 \%$, due to two cases of ineffectual catheters that were removed immediately after surgery. Re-siting of the catheters was not considered as it should only be performed under general anaesthesia. Even though five other patients received opioids during surgery, epidural analgesia was considered successful because it provided analgesia in the postoperative period. The secondary success rate of epidural analgesia was $66.7 \%$; the catheters were useful for analgesia until their planned removal, or earlier when the patient's postoperative recovery was better than expected. In some patients, epidural analgesia was considered successful despite being discontinued earlier than preoperatively planned, as it allowed the patient to avoid a prolonged stay in the HDU solely to manage the epidural analgesia.

Active management of epidural analgesia was necessary, with perioperative epidural top-ups and/or epidural infusion dosage adjustments to optimise analgesia. Only 14 catheters (28\%) did not require intervention or premature removal due to complications. The need for top-ups or rescue medication did not indicate epidural failure but the need for continuous assessment due to the dynamic nature of pain. This may also reflect the difficulty in assessing pain in this group of patients, as suggested by the proliferation of infant pain scales and the lack of a gold standard. Other than observing autonomic changes during surgical stimulation and using the FLACC scale, pain assessment was heavily dependent on the experience of the anaesthetist, adding to the complexity of infant pain management.

Infant epidurals are daunting because of the small margin of error in catheter insertion and pharmacological dose selection. The median depth of the epidural space in the present study was $11 \mathrm{~mm}$. The 'give' of the ligamentum flavum is harder to detect in infants due to the higher extracellular fluid content. There are also pharmacological considerations, including the patients' immature renal and liver functions, and their lower level of plasma-binding proteins, resulting in the potential for toxicity and a more permeable blood-brain barrier to local anaesthetics. The first signs of toxicity in infants are usually severe, such as convulsions, arrhythmias or cardiorespiratory arrest. Our department's drug dosage protocol is similar to those in international recommendations. ${ }^{(4)}$ An optimal catheter tip position enables relatively low doses of anaesthetic solution to achieve the desired effect. Despite evidence showing the lower success rate of the lumbar approach in inserting epidural catheters to the thoracic level, this technique is still used. ${ }^{(5)}$ Due to our small study population, we could not reliably show whether failure rates or the incidence of breakthrough pain was higher with the lumbar approach. The institution does not routinely use epidurograms to confirm the epidural catheter tip position due to the risk of radiation.

Our results suggest that the use of fentanyl as an adjunct in epidural analgesia reduces the likelihood of postoperative breakthrough pain. This is supported by a randomised trial, which showed that the addition of $2 \mathrm{mcg} / \mathrm{mL}$ fentanyl to epidural bupivacaine improved post-thoracotomy pain in infants without any increase in side effects. ${ }^{(6)}$ Opioids bind to the pre- and postsynaptic receptors in the dorsal horn, modulate nociceptive input and have a local-anaesthetic sparing effect.

The complications in these cases were mainly technical problems resulting in the premature discontinuation of epidural analgesia; $26 \%$ of the catheters were removed prematurely due to catheter disconnection, leakage or obstruction. This figure is high compared to an adult case series in which Bouman et al reported a $13.3 \%$ incidence of technical problems resulting in premature epidural catheter removal. ${ }^{(7)}$ Catheter disconnection was the most common cause in our case series although Luer lock connectors were used between the epidural catheter, filter, infusion tubing and drug syringe. In our institution, most catheter disconnections were not witnessed and the epidural catheters were subsequently removed. Ways to mitigate this problem include limiting the number of connection points or strengthening them with adhesives. Catheter leakage at the skin exit site also occurred due to the disparity between the size of the Tuohy needle and that of the catheter used for the catheter-throughneedle technique. Tissue glue or subcutaneous tunnelling may be useful in preventing leakage. ${ }^{(8)}$

Although postoperative fever in children is common and only a very small proportion of cases are associated with a septic process ${ }^{(9)}$ epidural catheter-related infections can be devastating 
and hard to diagnose or treat. ${ }^{(10)}$ The features of neuraxial infections can also be difficult to detect. Hence, sterile insertion techniques should be emphasised and vigilant postoperative monitoring is necessary. As the rate of catheter colonisation by bacteria is higher in caudal catheters, we prefer the thoracic approach to epidural analgesia. ${ }^{(11,12)}$

To the best of our knowledge, there are few studies on epidural analgesia in young children. Earlier studies of neuraxial techniques used on infants arose from a need to avoid general anaesthesia in high-risk groups. Bösenberg described the largest series of 211 lumbar and 29 thoracic epidural catheters in infants undergoing major surgery. ${ }^{(13)}$ The study reported good intraoperative analgesia with satisfactory analgesia in the postoperative period, with the majority managed by intermittent bupivacaine top-ups. Complications included one dural puncture, one seizure after bolus top-up, and 15 cases of bradycardia that required treatment with anticholinergics. Postoperative management and outcomes of the epidural analgesia were not discussed.

It is necessary to carefully select patients to maximise the benefit-risk ratio. In our institution, healthy infants who underwent major abdominal surgeries with significant postoperative pain were considered for epidural analgesia. Factors that helped the institution to achieve good outcomes included ensuring that epidural catheterisation was only performed by specialist paediatric anaesthetists and its practice of twice-daily assessments by a consultant-led acute pain service, close monitoring in the $\mathrm{HDU}$, active management of the catheters and multidisciplinary, patient-centred care. In such a setting, epidural analgesia provided good pain relief and facilitated postoperative tracheal extubation without significant complications.

As the present study was retrospective and covered a 14-year period, there was data from only a small number of epidural catheters. In addition to the known flaws of retrospective studies, the primary success rate in our cohort may also have been overestimated, as epidural catheterisations that were attempted but unsuccessful might have been missed. We defined successful epidural analgesia through clinical observations of pain relief, which was the current standard of care. However, there are alternative methods, such as using electrocardiograph signals or nerve stimulation guidance to locate the catheter tip position. ${ }^{(14,15)}$ Compared to a case series by Shenkman et al on low-weight infants, we did not experience any major complications such as apnoea, bradycardia, reintubation or catheter site infection. ${ }^{(16)}$ This was likely due to the better health of our cohort and the institution's use of short-acting opioid fentanyl only in infants aged $>6$ months. Complications might also be underestimated in the study, especially minor complications. Although patients were discharged from acute pain service follow-up on the day after the termination of epidural analgesia, we may need to look into establishing a formal longer-term follow-up system to assess for delayed complications.

In conclusion, our data suggests that in experienced hands, specialised settings and active management, the success rate of epidural analgesia in infants undergoing major abdominal surgeries is high and without major incident.

\section{REFERENCES}

1. Block BM, Liu SS, Rowlingson AJ, Cowan AR, Cowan JA Jr, Wu CL. Efficacy of postoperative epidural analgesia: a meta-analysis. JAMA 2003; 290:2455-63.

2. Goeller JK, Bhalla T, Tobias JD. Combined use of neuraxial and general anaesthesia during major abdominal procedures in neonates and infants. Paediatr Anaesth 2014; 24:553-60.

3. Merkel SI, Voepel-Lewis T, Shayevitz JR, Malviya S. The FLACC: a behavioral scale for scoring postoperative pain in young children. Pediatr Nurs 1997; 23:293-7.

4. Lönnqvist PA, Morton NS. Postoperative analgesia in infants and children. Br J Anaesth 2005; 95:59-68.

5. Blanco D, Llamazares J, Rincón R, Ortiz M, Vidal F. Thoracic epidural anaesthesia via the lumbar approach in infants and children. Anesthesiology 1996; 84:1312-6.

6. Ganesh A, Adzick NS, Foster T, Cucchiaro G. Efficacy of addition of fentanyl to epidural bupivacaine on postoperative analgesia after thoracotomy for lung resection in infants. Anesthesiology 2008; 109:890-4.

7. Bouman EA, Gramke HF, Wetzel N, et al. Evaluation of two different epidural catheters in clinical practice. narrowing down the incidence of paresthesia! Acta Anaesthesiol Belg 2007; 58:101-5.

8. Ilfeld BM. Continuous peripheral nerve blocks: a review of the published evidence. Anesth Analg 2011; 113:904-25

9. Yeung RS, Buck JR, Filler RM. The significance of fever following operations in children. J Pediatr Surg 1982; 17:347-9.

10. Seth N, Macqueen S, Howard RF. Clinical signs of infection during continuous postoperative epidural analgesia in children: the value of catheter tip culture. Paediatr Anaesth 2004; 14:996-1000.

11. McNeely JK, Trentadue NC, Rusy LM, Farber NE. Culture of bacteria from lumbar and caudal epidural catheters used for postoperative analgesia in children. Reg Anesth 1997; 22:428-31.

12. Bubeck J, Boos K, Krause H, Thies KC. Subcutaneous tunneling of caudal catheters reduces the rate of bacterial colonization to that of lumbar epidural catheters. Anesth Analg 2004; 99:689-93.

13. Bösenberg AT. Epidural analgesia for major neonatal surgery. Paediatr Anaesth 1998; 8:479-83.

14. Tsui BC, Wagner A, Cave D, Kearney R. Thoracic and lumbar epidural analgesia via the caudal approach using electrical stimulation guidance in paediatric patients: a review of 289 patients. Anesthesiology 2004; 100:683-9.

15. Tsui BC, Seal R, Koller J. Thoracic epidural catheter placement via the caudal approach in infants by using electrocardiographic guidance. Anesth Analg 2002; 95:326-30.

16. Shenkman Z, Hoppenstein D, Erez I, Dolfin T, Freud E. Continuous lumbar/ thoracic epidural analgesia in low-weight paediatric surgical patients: practical aspects and pitfalls. Pediatr Surg Int 2009; 25:623-34. 